\title{
La critica a la causalidad en T. W. Adorno: un propuesta filosófico-musical a través de Atmosphères, de Ligeti
}

The critique of causality in T. W. Adorno: a philosophicalmusical proposal through Atmosphères, by Ligeti

Marina Hervás Muñoz

Akademie der Künste (Berlín) mhermu@gmail.com

DOI: http://doi.org/10.15366/bp2019.21.009

Bajo Palabra. II Época. No21. Pgs: 159-174 


\section{Resumen}

Este artículo está dividido en dos partes: por un lado, se delinean los puntos fundamentales de la crítica a la causalidad de T. W. Adorno y, en especial, en referencia a Aristóteles y Kant. Por otro, se explicita la deriva de esta crítica en su propio proyecto filosófico, que presta especial atención, en sus últimos años, a la música de los jóvenes compositores de Darmstadt. Atmosphères, de Ligeti se constituye como una de las obras fundamentales para dar cuenta del cruce entre filosofía y música en el pensamiento adorniano y, en concreto, con respecto al problema de la causalidad.

Palabras Clave: T. W. Adorno, Ligeti, causalidad, Kant.

\section{Abstract}

This article has two parts: on the one hand, it attempts to outline the fundamental points of T. W. Adorno's critique of causality and, in particular, in reference to Aristotle and Kant. On the other hand, it made explicit the drift of this criticism in his own philosophical project, which pays special attention, in his late thoughts, to the music of the young composers of Darmstadt. Ligeti's Atmosphères is one of the fundamental works to account for the crossover between philosophy and music in Adornian thought and, in particular, with regard to the problem of causality.

Keywords: T. W. Adorno, Ligeti, causality, Kant. 


\section{Introducción}

LA TENSIÓN ENTRE LA CAUSALIDAD y el determinismo es un tema que protagoniza el siglo XIX y, especialmente, su relación con la aproximación científica a la realidad. Como muestra Kern ${ }^{1}$, sin embargo, no sólo hubo una tendencia en las ciencias naturales a articular un discurso coherente en torno a las leyes de la naturaleza, es decir, su articulación causal, sino también a derivar de ellas patrones de comportamiento social. El desarrollo del positivismo, gracias al Curso de Filosofía Positiva de Comte de 1830, entre otros, influye en esta tendencia que trató de secularizar la causa última de la acción de la divinidad. Las teorías sobre la determinación se construyeron bajo el supuesto de que la realidad está organizada según una legalidad que puede ser analizada y generalizada a partir de los meros hechos. Por lo tanto, la tarea principal de cualquier teoría sería encontrar los principios que estructuran secretamente la realidad. Pero el determinismo radical tiene sus consecuencias morales: si el principio último de una acción puede ser analizado tarde o temprano, la responsabilidad de cualquier acción estaría indudablemente en este principio último. Asimismo, sería posible articular toda la secuencia que conduciría a cualquier hecho futuro. De hecho, la exactitud de las predicciones comenzó a ser una de las principales cualidades de las ciencias naturales en contraste con las ciencias sociales o las humanidades.

La causalidad parece ser un tema secundario en la producción de Theodor W. Adorno, ya que no hay un solo texto que preste atención específicamente sobre este tema en su producción. Sin embargo, aparece constantemente en todas sus conferencias tardías y juega un papel relevante, al menos, en sus escritos tardíos, y prominentemente en la Dialéctica Negativa. En este artículo se mostrarán las coordenadas principales de este problema en relación al giro que, tal y como mostraremos, parece que experimenta su pensamiento con respecto a la música a partir de los años sesenta. La causalidad era un elemento crucial de su enfoque de un modelo filosófico sociopolítico, como ya lo han demostrado muchos estudiosos. Lo que afirmo es que desarrolló este concepto no sólo por medios puramente filosóficos,

${ }^{1}$ Kern, S., A Cultural History of Causality. Science, Murder Novels, and Systems of Thought. New jersey, Princeton University Press, 2004, 
sino teniendo en cuenta algunos de los principales problemas a los que se enfrentaron los compositores en estos años. En resumen, en este trabajo se explicaría cómo la atención de Adorno a la nueva música de los años 60 afectó profundamente a su filosofía y específicamente al problema de la causalidad.

El proyecto de Adorno se construye en torno a la crítica contra la prima philosophia. En sus ojos, en definitiva, hay una tendencia en la filosofía tradicional pero también en algunas de las propuestas del siglo XX, a buscar un primer elemento que sirva de base para el resto de la construcción filosófica. Por lo tanto, la mayoría de los proyectos filosóficos occidentales operan de manera deductiva. Precisamente, Adorno critica esta tendencia analizando qué concepción del tiempo está detrás de estos modelos. Según él, hay una primacía de una construcción lineal del tiempo. Esta construcción implica una organización del pensamiento que se basa en la correlación de eventos, en su mayoría organizados de acuerdo a una relación causal entre ellos.

Hay dos posiciones principales con respecto a la causalidad que Adorno analiza críticamente. En primer lugar, una que asume que una relación causal no existe realmente, sino que el sujeto atribuye al objeto un mecanismo de pensamiento como si fuera una característica del objeto mismo. La organización causal de los fenómenos, derivada de la temporalidad lineal, se observa entonces como si de hecho fuera idiosincrásica del objeto mismo. A través de esta estrategia, según Adorno, hay una repetición del sujeto en el objeto. Cuando se trata de conocer el objeto, el sujeto simplemente se encuentra en él. Los elementos cualitativos del objeto que no encajan con las exigencias del pensamiento causal son borrados o simplemente obviados. Habría, pues, una relación violenta con el objeto. Se le impone una unidad heterónoma, para propiciar así la coincidencia entre sujeto y objeto. Por lo tanto, el modelo filosófico que propone Adorno tiene que reformularse desde la crítica al pensamiento "causal-mecánico"[kausalmechanisches Denken]. En segundo lugar, por el contrario, la relación causal se entiende como existente en los objetos mismos. Esto se deriva de la concepción animista según la cual las cosas "tienen un alma interior y una determinación interior" ${ }^{2}$. Esta forma de pensar muestra la gran disparidad entre los desarrollos científicos y la "conciencia cotidiana". Su enfoque trata de abordar ambas posiciones en la filosofía tradicional y ofrecer no una respuesta cerrada a sus problemas, sino al menos cambiar la perspectiva desde la que ambos se enfrentan.

2 Adorno, T. W. Problemen der Moralphilosophie, Nachgelassene Schriften IV/10, Frankfurt a. M., Suhrkamp, 1996, p. 78 


\section{Revisión del problema de la causalidad en la filosofía tradicional}

Adorno trata de rastrear el problema de la causalidad en algunos de los filósofos más prominentes de toda la historia de la filosofía. No pretende hacer un estudio exhaustivo del problema, sino que fundamentalmente se concentra en dos posiciones al respecto. Trata del concepto aristotélico de causalidad ya que lo trata desde una perspectiva objetiva, esto es, desde el concepto de la materia[Stoff]; y del kantiano ya que, en principio, se articula como una categoría subjetiva, como ya en Hume.

El concepto de causalidad en Aristóteles, según la interpretación de Adorno, se deriva de una forma secularizada de la necesidad natural. Por lo tanto, causalidad en Aristóteles significa "causalidad natural" ${ }^{3}$. Aristóteles parte de un concepto secularizado de naturaleza en la que cada ser tiene una dirección determinada (telos). Esta característica dinámica de los seres implica un momento de arbitrariedad, ya que o bien se presupone que el ser se mueve a sí mismo (autómata), que comenzó a moverse en algún momento, dando así comienzo a un proceso casual; o bien solo cabe articular el movimiento desde el mero týche, es decir, desde el "azar". Sin embargo, hay una tensión que Aristóteles no resuelve. Consiste en la explicación de por qué hay elementos que obedecen a la organización teleológica de la naturaleza y a su aplicación en la sociedad; y otros que, sin embargo, parecen poder escapar de la teología. Para Adorno, esta tensión funda el núcleo mismo de la metafísica occidental, ya que se delinea uno de sus principales problemas, a saber, el choque aporético entre causalidad y libertad.

Así es como Adorno conecta a Aristóteles con Kant y, en concreto, con la tercera antinomia del crítico de la razón pura. La interpretación de Adorno de estas reflexiones antitéticas tiene que ver con un gesto de exageración de la razón ingenua. Kant asume primero que la causalidad real es una tendencia de los objetos mismos. Según Adorno, en la $K r V$ este enfoque científico natural se da por sentado. Por lo tanto, la causalidad se entiende principalmente, como en Aristóteles, desde la perspectiva de las ciencias naturales. Él, entonces, lleva esta suposición a sus últimas consecuencias para analizar las contradicciones que aparecen en ese proceso. En Kant la causalidad se definiría mediante el concepto de necesidad. Las relaciones causales podrían articular fenómenos arbitrariamente. Sin embargo, dado que Kant incluye la causalidad dentro de las categorías a priori, esto es, como condición del conocimiento, que la causalidad se hace necesaria. En este sentido, Adorno caracteriza la concepción de

${ }^{3}$ Adorno, T. W., Metaphysik. Begriff und Probleme, Nachgelassene Schriften IV/14, Frankfurt a. M., Suhrkamp, 1998, p. 118. 
Kant de la causalidad como circular. La necesidad es determinada por la causalidad; y la causalidad es considerada como legalidad [Gesetzmäßigkeit], es decir, como un evento organizado en cadena que sólo puede ser entendido de acuerdo a su estructuración previa. Otro elemento se añade a esta circularidad: la universalidad [Allgemeinheit]. Causalidad es, entonces, la capacidad de asociar dos eventos separados temporalmente en una unidad de saber. Es un proceso sintético, en el cual cual, los eventos individualmente separados temporalmente, se relacionan en una cadena casual los eventos individuales aparentemente separados temporalmente que se relacionan en una cadena casual. Las cosas en sí mismas no están organizadas de acuerdo con las relaciones causales, pero la causalidad es un medio de organizar el conocimiento sobre esas cosas. Por lo tanto, en el momento en que las reglas de esta organización se aplican a las cosas, hay un principio de generalización y necesidad en la estructura del conocimiento. Hay, no obstante, una aparente contradicción en la estructura temporal de la triada causalidad-necesidad-generalidad. Mientras que la causalidad se caracteriza por la sucesión en términos temporales, la generalidad se articula por la simultaneidad ${ }^{4}$, ya que se aplica a todos y cada uno de los eventos sin importar cuándo. La necesidad implica ambas estructuras temporales.

En el proyecto de Kant la causalidad es una de las categorías que garantiza la unidad de la razón. Por eso, para Adorno, la causalidad se vuelve ontológica en él 5 . El papel que juega la causalidad como categoría del entendimiento para articular el conocimiento como unidad no es, para Adorno, una categoría que se impone a la cosa, sino que sigue la estructura de la cosa misma, acudiendo así a otro pensamiento circular: "La unidad de conciencia en sí misma se presenta según el modelo de la unidad de la cosa. Aunque la cosa está constituida por la unidad de conciencia, requiere que haya algo así como una unidad de la cosa en general" 6 .

La legalidad que implica la causalidad, significa que cada desvío, cada variación, ya está regulada por una ley general. No hay ninguna posibilidad de modificar lo que ya está concebido en la cadena causal: cada engranaje está acoplado con precisión. Este concepto de causalidad se hereda de alguna manera de una manera mecanicista de pensar. De ahí que Kant se vea abocado a derivar la libertad de la causalidad. En palabras de Adorno, la libertad sería en realidad una especie de causalidad sui generis ${ }^{7}$. Como se señaló anteriormente, el conocimiento no es posible en absoluto sin la legalidad, sin la unidad que surge de las formas y categorías a

\footnotetext{
${ }^{4}$ Adorno, T. W., Kants „Kritik der reinen Vernunft", 1995, p. 214

${ }^{5}$ Ibid., p. 141.

${ }^{6}$ Ibid. 162.

7 Adorno, T. W. Problemen der Moralphilosophie, Nachgelassene Schriften IV/10, Frankfurt a. M., Suhrkamp, 1996, p. 63
} 
priori. La causalidad es una de las categorías que organizan el fenómeno puro. La libertad, por lo tanto, está sujeta a la articulación previa de las categorías, incluida la causalidad.

Adorno exagera metodológicamente las afirmaciones de Kant para extraer las implicaciones radicales de su teoría de la causalidad:

"Asumamos primero que el determinismo de la naturaleza es total, es decir, que todo en la naturaleza está determinado por causa y efecto, de conformidad con las leyes. En ese caso, la afirmación de Kant de que la ley moral es un hecho, una ordenanza, algo que se nos impone irresistiblemente, sería [...] profundamente inmoral. Sería inmoral porque plantearía exigencias a las personas que, por ser seres empíricos, no podrían satisfacer. [...] A la inversa, sin embargo, si los sujetos empíricos realmente pueden actuar libremente, entonces porque ellos mismos son parte de la naturaleza, la unidad kantiana de la naturaleza, fundada en las categorías, será destruida. La naturaleza tendrá entonces una brecha, y esta brecha violará la unidad de nuestro conocimiento de la naturaleza a la que, según Kant, aspiran las ciencias naturales" 8

Kant cae en la identidad entre libertad y legalidad mediante la unidad de la razón. Para él, cuanto más precisamente se articula la razón a través de las leyes, más libre es el sujeto. Eso implica, entonces, que la libertad sería un acomodo preciso entre la legalidad y la acción. La libertad se entiende en Kant, pues, como una autonomía radical, es decir, el grado máximo en que la razón se determina a sí misma.

\section{La causalidad en el proyecto filosófico adorniano}

Su propio proyecto parte de la premisa de que la causalidad y el azar [Zufall] están íntimamente relacionados, ya que la subsunción total bajo la causalidad es artificial. No hay, para él, ningún límite en la aparición de nuevas cadenas causales, ni siquiera en el análisis de ejemplos aparentemente simples para explicar la operatividad de la causalidad. Utiliza el ejemplo de dejar caer un libro. Aun cuando el análisis causal señalará que el libro se sintió caído (C) porque alguien lo dejó caer (A) y la gravedad (B), para Adorno hay otras cadenas causales que cruzan esta relacionada con el libro, y otras nuevas que se abren en el momento en que alguien decide dejar caer un libro en este contexto espacio-temporal.

El llamado "miedo al caos" es, para Adorno, una de las limitaciones más importantes del conocimiento. Su proyecto se basa en la crítica a la "prima philo-

${ }^{8}$ Ibid., pp. 150-151. 
sophia", el intento de establecer un elemento que sirva como fundamento de un proyecto filosófico (como la duda cartesiana) o como su principio último (como el Ser en el pensamiento de Heidegger). Toda la cadena causal está orientada a alcanzar el último punto o se sedimenta en una causa primordial. La contradicción en la que incurre el concepto de causalidad consiste en el problema de que esta primera o última causa no siempre puede determinarse y no podría cumplir con los criterios de universalidad; y, al mismo tiempo, sin ella, como se ha dicho, la cadena causal no tendría ninguna organización jerárquica y, por lo tanto, sería arbitrariamente estructurada. Para Adorno, no es necesario renunciar a este modelo del primer o último elemento. Adorno afirma que el elemento fundador, en lugar de ser "inmediato", "positivo" y "absoluto", es sólo un momento. Para él, existe una mediación mutua entre causa y efecto. La causa no puede ser pensada sin su efecto, en el sentido de que lo que se ha convertido ya afecta a la causa original.

Adorno es consciente de que la crítica a la causalidad puede llevar al problema del absurdo. La estructuración causal se aplica a muchos conceptos importantes que organizan la vida social, como la historia. Para Adorno, la atribución de un sentido a la historia deriva del sentido interiorizado de la vida misma. Este sentido de la vida misma, por su parte, deriva de la idea religiosa de que existe cualquier entidad que garantice que la vida individual es significativa y no sólo una existencia aleatoria. Según Adorno, la construcción significativa de estos elementos articuladores de la sociedad tiene como antecedente propio el pensamiento "causal-mecánico" [Kausal-mechaniches Denken], en el que se basa, por ejemplo, el concepto occidental de racionalidad. Por lo tanto, por ejemplo, se supone que hay una continuidad en la construcción del conocimiento.

Adorno entonces rechaza esta atribución de las leyes científicas de la naturaleza a los "procesos espirituales". Mientras que en ciencias los acontecimientos se estructuran según el modelo "siempre cuando- entonces" [immer wenn-dann], en ciencias sociales tiene que ser "después de -entonces"[nachdem-so]. Esto implica que, según él, en los procesos espirituales no hay leyes fundantes, sino tendencias y líneas de desarrollo. Por tanto, la relación entre causa y efecto no es la única estrategia teórica para analizar los eventos sociales. Podría haber algunos aspectos de ellos que no se pueden derivar en absoluto de una causa específica o que la causa está oculta o no está clara. Lo que Adorno añade, de alguna manera siguiendo a Bloch y Marcuse, es la dimensión utópica. La cuestión del análisis crítico consiste en la exploración de los procesos que están operando en la creación de narrativas (causales) y también en exponer qué otras realidades serían posibles: "Toda reificación es olvido, y la crítica significa en realidad lo mismo que el recuerdo, es decir, movilizar en fenómenos 
aquello por lo que se han convertido, reconociendo así la posibilidad de que se hayan convertido, y por lo tanto puedan ser, algo diferente" "

En definitiva, en el proyecto de Adorno se parte de la crítica a la consideración del objeto como una totalidad que debe o incluso puede ser comprendida. En sus palabras, "nada en el mundo está compuesto... de hecho y de concepto" ${ }^{10}$. Por lo tanto, Adorno rechaza que la tarea de la teoría sea, tal como lo plantean los positivismos, la mera descripción de los hechos; o, como en la mayoría de los enfoques sociológicos, delinear las tendencias sociales. Para él, tales tendencias no son más que una "extrapolación y generalización" de algunos hallazgos del conjunto social, que permanecen en el plano de los hechos. El concepto de teoría en Adorno busca lo no idéntico, superar la mera repetición o descripción de lo dado, es decir, debería acudir a lo que no está ya meramente presentado en los hechos. La teoría tiene que aspirar a lo radicalmente otro. Adorno encuentra en Kant, pero también en otros pensadores, como Hegel, la tendencia a organizar la experiencia a través de la teoría filosófica. Lo que Adorno intenta, por el contrario, es delinear lo que podría ser una experiencia no reglada [unreglementierte Erfahrung]. Este tipo de experiencia parte de una crítica radical a la autonomía de la razón y a su identidad con la libertad en Kant. La razón, por el contrario, no satisface la demanda de un compendio preciso y estricto de leyes, sino, por el contrario, muestra desde sus aporías todo lo que es reprimido por ella misma. En sus análisis, Adorno intenta mostrar cómo la filosofía tradicional une la libertad y la praxis represiva. Se niega la libertad para no enfrentarse a las contradicciones que caracterizaron a la realidad y a la sociedad. La estructuración de la experiencia bajo el reino de la causalidad implica que la acción humana se limita al análisis de la estructura acción-reacción.

La lógica tradicional tiende a clasificar bajo conceptos generales, a partir de los cuales los objetos se constituyen como tales o, en otras palabras, dejan de ser amorfos. Adorno, entonces, busca explorar la posibilidad de pensar fuera del alcance de la lógica tradicional. En otras palabras, lo que Adorno busca es desentrañar la correlación artificial entre estructura de pensamiento y estructura del ser, algo que él considera como una hipóstasis de la lógica.

Adorno encuentra en el arte un lugar privilegiado para reflexionar sobre estos asuntos. A su juicio, el arte no responde en absoluto a la cadena causal, ya que el objeto artístico siempre va más allá del propio concepto de arte. Adorno no sugiere, entonces, depurar este concepto hasta que se adapte a cualquier tipo de obra de arte, sino asumir que el arte mismo no puede obedecer a los medios de

\footnotetext{
9 Adorno, T. W., Einleitung in die Soziologie. Nachgelassene Schriften IV/15, Frankfurt a. M., Suhrkamp, 1993, p. 150.

${ }^{10}$ Adorno, T. W., Dialéctica negativa, Madrid, Taurus, 1984, p. 189 (traducción modificada).
} 
conceptualización. Esta estructura no conceptual del arte, junto con la consideración del espíritu o creador como un momento (y no como el punto de partida), implica que no existe una construcción causa-efecto en el arte. La estética debe abordar, según Adorno, la negación radical de la comprensibilidad, la adscripción inteligible a la obra de arte. En otras palabras, el arte no entra dentro de la lógica que tradicionalmente se atribuye al pensamiento conceptual. Adorno, por ejemplo, señala la "alogicidad" de una fuga de Bach. Incluso cuando se considera como una forma lógica, ya que tiene sujeto, contra-tema y respuestas, así como se divide en exposición, sección media y sección final, necesariamente para ser una fuga, no hay manera de encontrar una estructura lógica en términos de premisas y conclusiones ${ }^{11}$. La necesidad de la organización de los materiales está en cada obra planteada desde cero. No hay subsunción bajo un universal [eine Allgemenheit], a diferencia del pensamiento lógico tradicional.

\section{Adorno y Ligeti}

En el AÑo 1957, el joven musicólogo Heinz-Klaus Metzger hizo una crítica a Th. W. Adorno que, como se muestra en otro lugar ${ }^{12}$, marcó en cierto modo los derroteros del pensamiento musical -y como creo que no pensaba la filosofía sin la música, también musical- en su última década de producción. La crítica apareció en un artículo que unía el título de Filosofía de la nueva música, libro de referencia a partir de su aparición para los nuevos compositores y "Das Altern der neuen Musik" (1954), adquiriendo el nombre de "El envejecimiento de la filosofía de la nueva música" ${ }^{13}$. En esta crítica, fundamentalmente, Metzger constata cómo muchas de las ideas que habían venido articulando el pensamiento filosófico-musical de Adorno han dejado de dar cuenta de los nuevos procesos compositivos, especialmente los desarrollados en torno a los Cursos Internacionales de música de Darmstadt. El propio Adorno, poco a poco, da la razón a Matzger y se dispone, en sus últimos años de producción, a intentar repensar tales ideas. Aunque son varios compositores los que le despiertan interés de forma explícita, como Boulez o Stockhausen, en este caso me centraré en las reflexiones sobre Atmosphères, de Ligeti, como ejemplo fundamental para entender esta crítica a la causalidad en Adorno.

${ }^{11}$ Lecciones de Estética de 1961, que se pueden encontrar en el Archivo de Adorno de Frankfurt a. M. La referencia se encuentra bajo la signatura Vo 6582, del 27 de julio de 1961.

12 Hervás Muñoz, Marina, Pensar con los oídos: conocimiento y música en la filosofía de T. W. Adorno, Tesis doctoral. Barcelona, Universitat Autònoma de Barcelona, 2017.

13 Metzger, H-K., "Das Altern der Philosophie der neuen Musik”, en Die Reihe, vol. 4 (1958), pp. 64-80. 
Ligeti y Adorno coinciden temporalmente en su consideración crítica sobre la causalidad. Tal y como constata Floros, a finales de los ańos 60 Ligeti expresó explícitamente la fuerte influencia de Alicia en el pais de las maravillas y Alicia a través del espejo, de Lewis Carroll, para sus reflexiones sobre la causalidad, que tuvieron su reflejo musical en obras de aquellos años, como Adventures, su Cuarteto n. 2 o las Diez Bagatelas para Quinteto de viento, donde cita un pasaje de Alicia en el pais de las maravillas ${ }^{14}$. Es justamente, como hemos visto, este mismo periodo en el que Adorno se dedica más activamente a este problema, probablemente con el objetivo de articular un discurso sobre la libertad, algo que se ve tanto en su filosofía moral como en "Vers une musique informelle" (1961). No obstante, este asunto venía fraguándose en su pensamiento desde antes, al menos desde Minima Moralia. En una critica a Benjamin, Adorno señala que el filósofo berlinés cae en un dualismo al comprender la escritura de la historia desde la perspectiva de los vencidos y los vencedores. Para él, hay algo en la "desdichada linealidad" de la historia que no encaja exactamente en su constitución, que queda retirado como "material de desecho" y "punto ciego", "inesencial", "desplazado" y "grotesco": "libros infantiles como Atice in Wonderland o Struwwelpeter, ante los que la pregunta por el progreso o la reacción sería ridícula, contienen cifras de la historia incomparablemente más sugestivas que el gran teat ro monta do por Hebbel con la temática oficial de la culpa trágica, el cambio de los tiempos, el curso del mundo y el individuo» ${ }^{15}$. Ambos, por tanto, aluden a formas de constituir una narración sin la atadura de la linealidad y, sobre todo, sin la concatenación lógica, que puede derivar en la exageración (forma que Adorno reclama para sí), el doloroso absurdo de Beckett o la puesta a prueba de formas de construcción no causal, donde hay elementos emergentes y disruptivos.

Atmosphères (1961) es una obra pensada de forma textural, de tal forma que el entramado y las voces individuales quedan engarzadas, de tal modo que no se privilegian parámetros musicales tales como la melodía y la armonía, sino que la prioridad la tiene el color. El color, en estos años, también adquiere un lugar privilegiado en la filosofía adorniana. Al menos tres veces el uso y la función del color en la música fue analizada específicamente por Adorno. El primero es "Konstruktion und Farbe" [Construcción y color], dos sesiones de conferencias que dio en enero de 1959 para la NDR. Permanece inédito y se encuentra en el Adorno-Archiv de Berlín bajo la firma VT 140 y VT 141. La segunda es el resultado de la transcripción del ciclo de conferencias que impartió en el Curso Internacional de

\footnotetext{
${ }^{14}$ Floros, C., György Ligeti. Beyond Avant-garde and Postmodernism, Frankfurt a. M., Peter Lang, 2004, 20.

15 Adorno, T. W., Minima Moralia, Madrid, Taurus, 2001, p. 151.
} 
Darmstadt de $1966^{16}$. El primer documento es de alguna manera un resumen de las conferencias, con algunos fragmentos auténticos, o al menos la base para ellos. Hay otro documento que amplía algunas de las cuestiones elaboradas en los textos mencionados anteriormente. Este también es inédito y se refiere a dos conferencias que dio en el Conservatorio de Frankfurt (Frakfurter Musikhochschule) en 1961. Se llama "Funktion des Klanges in der neuen Musik" [Función del sonido en la nueva música] y también se encuentra en el Adorno-Archiv bajo la firma VT 168 y VT 169.

Más allá del asunto documental, es fundamental remarcar que el color adquiere un papel fundamental para entender la importancia de Ligeti y, en concreto, de esta obra, para la crítica de la causalidad. Y es que, Adorno, también en 1961, escribe "Über Statik und Dynamik als soziologische Kategorien"17, donde reconoce que hasta entonces, había considerado las categorías de "estático" y "dinámico" de formas no plenamente dialécticas. Dicho en breve, en este texto comienza a pensar la posibilidad de que lo dinámico solo se constituya asumiendo en él un momento estático y viceversa. Esta reflexión la extrapola al sonido como lo temporal (dinámico) y la imagen como lo espacial (lo estático). La obra de Ligeti pone esto en juego, suspendiendo la estructuración temporal lineal. La construcción en planos que, al mismo tiempo, avanza temporalmente, implicaría un intento de aproximar, a nivel sonoro, esta tensión dialéctica entre lo estático y lo dinámico.

La dificultad que encuentra Adorno es cómo compatibilizar -si es que posible aún tal compatibilidad- la línea de voces o, en sus términos, "das Desiderat der Deutlichkeit", lo que hace patente los elementos constructivos y separaba el primer plano del segundo plano y el trabajo sobre un único plano, o al menos no en términos de primero y segundo que propone la Klangfarbenmelodie ${ }^{18}$ schönbergiana Para él, ejemplo de esto aparece en Farben de las Fünf Orchesterstücke Op. 16, la cual relaciona con Atmosphères de Ligeti, en la que "la forma se constituye desde el

\footnotetext{
${ }^{16}$ Adorno, T. W., Kranichsteiner Vorlesungen, Nachgelassene Schriften, IV, 17, Berlin, Suhrkamp, 2014.

17 Adorno, T. W., Gesammelte Werke, Volumen 8, Frankfurt a. M., Suhrkamp. 1976, pp. 217-238.

18 Schönberg, A., Harmonielehre. Viena: Universal, 1922, p. 507. Hay una larga discusión sobre este concepto. Farben ha sido tomada por muchos intérpretes como el lugar donde Schönberg depsliega esa Klangfarbenmelodie. Sin embargo, Dahlhaus hace notar que el concepto de Klangfarbenmelodien es de 1911, considerado entonces por Schönberg como una "fantasía futura" [Zukunfstphantasie], mientras que Farben es de 1909. Dahlhaus, además, remarca la dificultad de definición del término, en la medida en que no queda muy claro si se trata de una suerte de "coloración" [Farbung] de una altura, si se trata de una igualación del timbre y de la altura (como parece que sugiere supra), de la diferencia de instrumentación de una altura o, como sugiere él, la invitación a desarrollar una lógica de los timbres, al igual que ya se ha articulado en otros parámetros musicales. Dahlhaus, C., "Schönbergs Orchesterstück op. 16, 3 und der Begriff der „Klangfarbenmelodie““", en VV. AA., Bericht über den Internationalen Musikwissenschaftlichen Kongress Bonn 1970, Kassel, Bärenreiter, 1971, pp. 372-373.
} 
color" ${ }^{19}$. Sin embargo, Ligeti no es, para Adorno, un compositor "tímbrico" [Klangfarbenkomponist], en la medida en que, para el húngaro, "el descubrimiento de nuevos timbres no era un fin en sí mismo, sino solo un medio de la configuración de la forma" ${ }^{20}$. Adorno, no obstante, no explora verdaderamente un concepto clave en Ligeti, el de textura, tejido [Gewebe], que permite pensar en la disposición de lo sonoro en un plano sin la necesidad de una construcción temporal lineal de continuum, como vemos en Atmosphères. Sin embargo, Adorno extrae de la reflexión sobre el color la consideración del sonido como exceso, como algo que no cabe en la "exacta fantasía", en la imaginación que termina siendo correspondida por la realidad. Hay algo que sobresale, que supera tal imaginación. El color es, en cierto modo, la marca de lo no absolutamente controlado, de lo inesperado para el oído.

Mediante la reflexión sobre el color, al igual que en "Vers une musique informelle", Adorno está tratando de pensar, desde la música, lo que en Dialéctica Negativa llama "lo añadido" [ Das Hinzutretende], lo que rompe con el esquema causa-efecto. En Atmosphères, la forma emerge de lo plástico, y no se le impone a lo sonoro desde fuera. Es decir, la forma es emergente desde la propia disposición del material, algo que Adorno exige en la teoría del conocimiento para el concepto, que no debería reducir la multiplicidad del objeto a lo que el concepto puede, sino tratar de dar cuenta su incapacidad para atraparlo. La experiencia ante tal forma emergente es, para Adorno, cifra de la experiencia no reglamentada, pues se constituye a la vez que el despliegue de la forma.

Mientras Adorno, en el marco de la Filosofía de la nueva música, seguía pensando en categorías de "antecedente" y "consecuente" o estructuras temporales como "expectativa-espera", a partir de su contacto con la "nueva generación" de compositores empieza a pensar desde otra perspectiva el tiempo, musical y filosóficamente. La causalidad, en lo musical, comienza a desvanecerse cuando se piensa en relación a lo que se le opone a lo sonoro, lo estático, tal y como se plantea en Atmosphères. Su constitución textural abre y disloca a la vez múltiples posibles cadenas causales, que quedan frustradas ante el entretejido del que parte. Lo que la obra muestra, a nivel sonoro, es la crítica al agotamiento de las formas de disposición del tiempo, así como la dimensión espacial -en este caso, en planos- de lo temporal, que también afecta a la constitución de lo causal, pues obvia la tensión dialéctica entre espacio y tiempo e impone una estructura temporal cerrada.

La definición clásica de causalidad implica, en definitiva, la conversión de una relación espacial de dos objetos en un objeto temporal: una relación entre A y B,

\footnotetext{
19 Adorno, T. W., Kranichsteiner Vorlesungen, Op. Cit, p. 511.

${ }^{20}$ Ibidem.
} 
que están situados en posiciones x e y, se convierte en temporal cuando B está allí por A, o A conduce a B en una estructura de temporalidad lineal, en la que A debe emerger antes que $\mathrm{B}$ para establecer tal relación. Por lo tanto, hay dos aspectos a modificar: primero, la relación no mediada entre el espacio y el tiempo que se supone establece la necesidad de causalidad; y segundo, la mera presunción de la estructura lineal del tiempo, que es también un requisito de las cadenas causales. Adorno, a mi juicio, en sus escritos tardíos, para pensar las relaciones espacio-temporales desde una perspectiva renovada, recurre a conceptos texturales, algo que se desarrolla gracias al cruce de las artes visuales y sonoras. Es evidente, gracias a textos como "Die Kunst und die Künste" (1966), que Adorno estaba atento al complejo diálogo entre las artes de las últimas décadas que aún continúa. El arte, en definitiva, no se puede pensar más como disciplinas separadas. No sólo afecta a la definición del arte en sí -sería mucho más acertado hablar de "arte" o de "proyectos artísticos"-, sino también a cada uno de los ámbitos a los que se supone que pertenece cada arte. La música, entonces, ya no era sólo un "arte temporal".

Como última observación hay que destacar que Adorno no anula radical y literalmente el problema de la causalidad. Su proyecto no entra dentro del concepto de "acto gratuito". Se trata, sin embargo, de subrayar la multiplicidad de temporalidades que actúan en el análisis de un hecho cualquiera y también la potencialidad de lo desconocido, es decir, lo que queda fuera de la mera estructuración del conocimiento según una construcción ideológica específica de lo que debe ser el conocimiento. Su crítica a la causalidad, entonces, se dirige a lo incognoscible. 


\section{ReferenCias Bibliográficas}

Adorno, T. W. Problemen der Moralphilosophie, Nachgelassene Schriften IV/10, Frankfurt a. M., Suhrkamp, 1996.

- Dialéctica negativa, Madrid, Taurus, 1984, p. 189 (traducción modificada).

- Einleitung in die Soziologie. Nachgelassene Schriften IV/15, Frankfurt a. M., Suhrkamp, 1993.

- Gesammelte Werke, Volumen 8, Frankfurt a. M., Suhrkamp. 1976, pp. 217-238.

- Kants „Kritik der reinen Vernunft“, Nachgelassene Schriften IV/4, Frankfurt a. M., Suhrkamp, 1995.

- Kranichsteiner Vorlesungen, Nachgelassene Schriften, IV/17, Berlin, Suhrkamp, 2014.

- Minima Moralia, Madrid, Taurus, 2001, p. 151.

- Lecciones de Estética de 1961, que se pueden encontrar en el Archivo de Adorno de Frankfurt a. M. La referencia se encuentra bajo la signatura Vo 6582, del 27 de julio de 1961.

- Metaphysik. Begriff und Probleme, Nachgelassene Schriften IV/14, Frankfurt a. M., Suhrkamp, 1998.

Floros, C., György Ligeti. Beyond Avant-garde and Postmodernism, Frankfurt a. M., Peter Lang, 2004, 20.

Hervás Muñoz, Marina, Pensar con los oídos: conocimiento y música en la filosofía de T. W. Adorno, Tesis doctoral. Barcelona, Universitat Autònoma de Barcelona, 2017.

Kern, S., A Cultural History of Causality. Science, Murder Novels, and Systems of Thought. New jersey, Princeton University Press, 2004,

Metzger, H-K., "Das Altern der Philosophie der neuen Musik", en Die Reihe, vol. 4 (1958), pp. 64-80.

Schönberg, A., Harmonielehre. Viena, Universal, 1922, p. 507.

VV. AA., Bericht über den Internationalen Musikwissenschaftlichen Kongress Bonn 1970, Kassel, Bärenreiter, 1971.

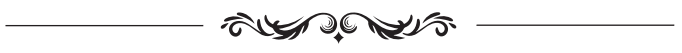

DOI: http://doi.org/10.15366/bp2019.21.009

Bajo Palabra. II Época. No21. Pgs: 159-174 
